\title{
Processing spatial information and the selective-masking effect
}

\author{
D. J. K. MEWHORT and A. J. CAMPBELL \\ Queen's University, Kingston, Ontario, Canada K7L 3N6
}

\begin{abstract}
When a mask follows tachistoscopic presentation of a letter string, the performance on the middle letters is reduced more than at the ends, the selective-masking effect. The endsfirst explanation for selective masking holds that the letter string is identified from the ends to the middle. As a result, the end items escape the effects of the mask. Using a bar-probe task, we present three kinds of evidence both questioning the ends-first account and offering an alternate based on spatial-localization processes. The first experiment exposed the role of spatial localization by using words to minimize identification factors. The second obtained a comparable selective-masking effect with pseudowords; again, the pattern of errors favored the localization account. A final experiment tested predictions derived from the localization view in response to inconsistencies posed by the sequential-identification idea.
\end{abstract}

When a character string is presented tachistoscopically and followed by a masking stimulus, the mask is not equally effective at all stimulus positions. Instead, the mask reduces report of the middle items more than that of the end items (Merikle, 1974; Merikle, Coltheart, \& Lowe, 1971). Merikle and his associates have documented selective masking under a variety of conditions and have proposed an explanation based on a sequential identification strategy. According to their account, subjects identify the characters of a string in an ends-to-middle order. As a result, the end items become available for report before the central ones. When a mask is introduced, it is relatively ineffective at the ends, because those items become available before the mask can intervene. Similarly, because the central items are last to be processed, they suffer the consequences of the mask to a greater extent.

Merikle and Coltheart (1972) used the ends-first idea to predict inverse selective masking in the forward-masking paradigm. Assuming that the effectiveness of a forward mask decays rapidly, the end items should be subjected to the full force of the decaying forward mask, but because they are processed later-when the mask has lost some of its effect-the middle items should be subjected to a less forceful disturbance. The results paralleled the prediction; a forward mask reduced report of the end items more than that of the middle ones.

\footnotetext{
The research was supported by a grant from the National Research Council of Canada (AP-318). We thank Pat Crawford for assistance in conducting the experiments. In addition, we thank B. E. Butler, G. T. Campbell, P. J. Hearty, G. D. Logan, and $B$. R. Shelton for comments and suggestions at various stages of the work. Address for reprints: D. J. K. Mewhort, Department of Psychology, Humphrey Hall, Queen's University, Kingston, Ontario, Canada K7L 3N6.
}

The convergence illustrated with forward masking would appear to put the ends-first idea on a solid footing. Nevertheless, Matthews (1973) has suggested an alternate explanation for the forward-masking result, and others have argued against the explanation for backward-masking examples. Using a financial incentive, for example, Henderson and Park (1973) altered subjects' processing strategy by rewarding performance differently at the ends and the middle; the selective-masking effect, however, remained stable. Because the effect remained stable across conditions involving different processing strategies, Henderson and Park argue that the endsfirst interpretation is wrong. In addition, there are a number of logical and empirical inconsistencies in the evidence thought to support the ends-first view. Merikle, Coltheart, and Lowe (1971), for example, reported two free-recall backward-masking experiments involving a manipulation of processing time over the range of 30 to $200 \mathrm{msec}$. Selective masking was found at each interval but, contrary to the endsfirst interpretation, was not affected by increases in time. According to the ends-first idea, the end items escape the effects of the mask because they are processed first. By the same logic, increasing the processing time should have permitted more interior items to escape the mask. Thus, while the free-recall experiments document the basic selective-masking effect, they also provide negative evidence for the ends-first interpretation.

Although they did not acknowledge the earlier failure of the predictions, Merikle and Glick (1976) tested the processing-time predictions in a bar-probe experiment. With minor exceptions to a strict interpretation, the predictions were confirmed, i.e., increasing processing time permitted interior items to escape the effects of the mask. Thus, the ends-first 
idea does not provide a general description of early identification processes, but it might apply in the probe paradigm.

The present studies question the ends-first idea by reexamining the evidence provided in bar-probe tasks. Our point of departure concerns the dependent measure (proportion of items reported correctly) and the interpretation associated with it. Typically, the proportion correct is taken to be an unbiased measure of the subjects' ability to identify the material; when subjects fail to report correctly, it is presumed that they have failed to identify the material correctly. The bar-probe task, however, requires more than simple identification; in addition to acquiring identity information, subjects must locate each item relative to the probe. Errors, therefore, may reflect failure of localization as well as of identification. Further, it seems reasonable to suppose that a mask has less effect on location information at the end positions, the natural spatial anchors. Thus, the selective-masking effect may well reflect difficulties in localization, not problems of identification.

Although the identification/localization distinction has been noted before (see Dick, 1974; Snyder, 1972), with the exception of Lowe's (1975) work, it has not been acknowledged by ends-first theorists. To some extent, acknowledging the distinction would change one of their basic theoretical preferences. As Eriksen and his colleagues have demonstrated (e.g., Eriksen \& Rohrbaugh, 1970), the distinction opens questions of selective attention, the use of various cues, and the independence of spatial and identity information. Ends-first theory attempts to finesse such questions by focusing on a sequential-identification process. Butler (1975) and Butler and Merikle (1973), for example, take the appearance of selective masking as an index of selective attention.

\section{EXPERIMENT 1}

Typically, the proportion of items reported correctly has been taken to be an unbiased index of subjects' ability to identify the material. Nevertheless, in a bar-probe task, the measure includes localization information. The first experiment was designed to focus on the latter component in the context of the selective-masking effect. In the experiment, subjects were shown an eight-letter word followed by a bar probe indicating which letter to report. In addition, on half of the trials, the probe was accompanied by a mask covering the whole word. In a pilot study using the same exposure and masking conditions, subjects produced virtually no errors when required to name the words. ${ }^{1}$ Thus, the display parameters should ensure minimal difficulty in letter identification; the probe task retains, however, the localization problems associated with the paradigm. Accordingly, if the selective-masking effect is the result of identification processes, it should not appear in the present task. Alternately, if the effect reflects localization difficulties, a typical selective-masking effect should emerge.

\section{Method}

Subjects. The subjects were 16 students at Queen's University. All were volunteers and reported normal or corrected-to-normal vision.

Materials and apparatus. The stimuli were 160 eight-letter words. The words, taken from the word-frequency lists provided by Kucera and Francis (1967), are the most frequent eight-letter words which do not include repeated letters.

The stimuli were displayed on a Tektronix point-plot display monitor (Model 604) supplied with P4 phosphor and controlled by a minicomputer (PDP/8e). The monitor was located in a partially darkened room adjacent to the room housing the computer.

The words were shown in uppercase letters; each letter was defined in a 5 by 7 matrix. A mask character involved all 35 dots of the matrix. The monitor's screen subtended a visual angle of about $8^{\circ} 5^{\prime} \times 6^{\circ} 18^{\prime}$. The word was centered on the screen and subtended a visual angle of about $3^{\circ} 6^{\prime} \times 21^{\prime}$. A probe in the form of a short arrow was presented about $18^{\prime}$ above the center of one of the letters. At its greatest extent, the arrow was full character size $\left(21^{\prime} \times 15^{\prime}\right)$ and consisted of a single column of dots with two three-dot fins extending at $45^{\circ}$ and $135^{\circ}$ from the bottom of the column.

Each trial involved a series of displays. For each, the material was refreshed continuously. The maximum refresh speed was limited by the settle time of the cathode-ray tube. To avoid confounding display size with brightness, a null-character technique was used. The technique slows successive refresh cycles by pausing between cycles for smaller displays by the time required to make the brightness equivalent to that for larger displays (see Mewhort, Note 1, for a discussion of our display algorithm).

Procedure. On each trial, a central fixation dot appeared. When ready, the subject pushed a button to initiate the trial. The fixation dot was replaced by a word for $100 \mathrm{msec}$. Immediately following the word, a probe appeared for $150 \mathrm{msec}$ above one of the letters of the word. On half of the trials, a row of mask characters covering each letter position appeared concurrently with the probe.

After each trial, the screen remained blank for about $2 \mathrm{sec}$. During the blank interval, the display routine prepared the next trial. When ready, the routine displayed the fixation dot and waited for the subject to initiate the trial.

The subjects were required to report the letter indicated by the probe, and a response was required on each trial. Although they were not told the stimuli were words, the subjects commented on the fact within a few trials.

Before starting the experiment, each subject received 32 practice trials. The practice involved eight-letter pseudowords generated for the purpose. At the start of the practice, the pseudowords were presented for $400 \mathrm{msec}$; during the practice, the duration was reduced to $100 \mathrm{msec}$ in four 100 -msec steps.

Design. Each subject received 160 trials, 10 replicates for the factorial combination of two variables, masking and probe position. Each letter position was probed equally often, and the mask was present on half of the trials. Each word was shown once to each subject; across subjects, each word was probed equally often at all 16 combinations of masking and letter position. The order in which the conditions were administered was determined randomly for each subject.

\section{Results and Discussion}

The responses were scored by counting the number 
of correct reports along with the separate tallies for intrusion and inversion errors. An intrusion error occurs when subjects report a letter not shown in the stimulus; an inversion occurs when subjects report a letter from the display other than the one indicated by the probe.

Figure 1 shows the proportion of letters reported correctly as a function of probe position and masking. Overall, masking reduced accuracy of report, $F(1,15)$ $=17.15, \mathrm{p}<.001$. More important, the mask had greater effect on report from the middle of the array than on that from the end of the array. With no mask, accuracy across the array took the W shape characteristic of the bar-probe procedure. With the mask, however, performance took a U shape across the array. The differential effect of the mask produced a significant interaction of masking with probe position, $F(7,105)=6.89, p<.001$. In particular, the quadratic and quartic components of the interaction were significant, $F(1,15)=35.61, \mathrm{p}<.001$, and $F(1,15)=5.38, p<.05$, respectively.

As is clear in the figure, the accuracy of report data showed a typical selective-masking effect. Previously, such results have been taken as prima facie evidence for the ends-first theory. In the present case, however, the results provide evidence against the theory.

The ends-first idea holds that the mask has a greater effect in the center than at the ends because the middle items are identified later. The present case, however, minimized the contribution of letteridentification processes. That a typical selectivemasking effect emerged, then, suggests that the effect reflects spatial-addressing problems rather than letteridentification processes. Because the ends-first idea is an account of letter-identification processes, the present data provide negative evidence for the theory, i.e., they divorce the theory from its basic empirical support.

Intrusions were extremely rare; they accounted for $1.72 \%$ of the responses (8.64\% of the errors). Inversions, then, accounted for more than $90 \%$ of the errors, and the bulk of the inversions were letters adjacent to the item probed, $73 \%$ in the mask condition and $84 \%$ in the no-mask condition. Figure 1 shows the inversion errors as a function of masking and probe position. Inversions were the mirror image of the accuracy data; the pattern of errors shows that the limitation to performance concerns spatialaddressing rather than letter-identification processes.

\section{EXPERIMENT 2}

The first experiment exposed the contribution of localization processes by minimizing that of identification factors. Previous work on the selective-masking effect has used nonsense stimuli rather than words,

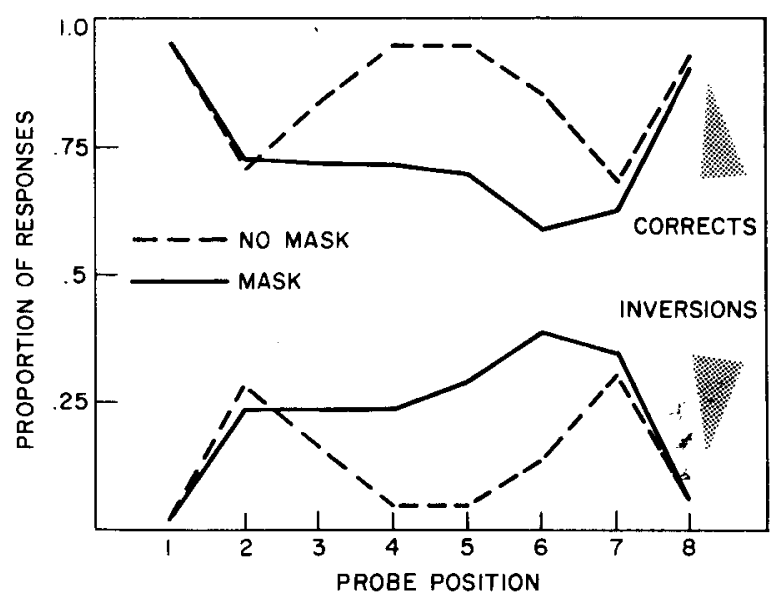

Figure 1. Proportion of both letters reported correctly and inversion errors in Experiment 1 as a function of probe position and masking.

and the localization factors revealed in a word experiment may provide a relatively small contribution to performance in such tasks. By using words to eliminate the contribution of identification factors, then, the first experiment may have inflated the relative importance of localization.

The second experiment, like the first one, questions the ends-first interpretation by focusing on localization factors. Unlike the first experiment, however, the stimuli were pseudowords of various orders of approximation to English. With such material, we cannot guarantee to minimize the contribution of identification processes. Instead, we rely on an analysis of errors to indicate what factors determine performance in the task.

\section{Method}

Subjects. The subjects were 16 undergraduates enrolled in introductory psychology courses at Queen's University. Participation fulfilled part of a course requirement. All subjects reported normal or corrected-to-normal vision, and none had participated in the previous study.

Apparatus and Materials. The stimuli were presented using the same apparatus as in the previous experiment. The materials were 128 pseudowords; 96 pseudowords were taken from the lists provided by Hirata and Bryden (1971, Table 6). The latter comprised 32 examples of zero-, second-, and fourth-order approximations to English. The remaining 32 pseudowords (called negative secondorder sequences) were constructed from the second-order material. Using the letter-frequency norms provided by Mayzner and Tresselt (1965), the position in the frequency table was noted for each letter in the second-order materials. The rank order of the frequency table was inverted, and the negative second-order sequences were generated by substituting letters in the second-order material with letters taken from corresponding positions in the inverse ranks. Thus, each second-order pseudoword had a corresponding sequence with equivalent sequential constraint but with the familiarity removed (see Garner, 1962, p. 255).

Procedure. At the start of each trial, a central fixation point was presented. When ready, the subject pushed a button to initiate the trial. The display, an eight-letter pseudoword, was presented 
for $150 \mathrm{msec}$. Immediately after the display, a probe appeared above one of the letter positions for $20 \mathrm{msec}$. On half of the trials, a mask covering all eight letter positions followed the probe; the mask duration was 150 msec. ${ }^{2}$

The instructions explained the nature of both the task and the materials. Thus, the subjects knew that some of the pseudowords might look like English but that none were misspelled or mutilated words. Further, the subjects were required to make a response after each trial. Before starting the experiment, the subjects were given the same practice trials as in Experiment 1.

Design. Each subject received 128 trials, two replicates for the factorial combination of three variables: position probed, masking, and approximation to English. Each pseudoword was used once with each subject; across subjects, each pseudoword was probed equally often at each stimulus position and masking combination. The order in which the conditions were administered was randomized independently for each subject.

\section{Results}

The responses were scored by counting the number of correct reports with separate tallies for intrusions and for inversions. The data are summarized in Figures 2 and 3. The top panel of Figure 2 shows both the proportion of letters reported correctly and the intrusions as a function of masking and probe position; the bottom panel shows the inversion errors as a function of masking and probe position. Figure 3 shows both intrusion errors and accuracy as a function of approximation to English.

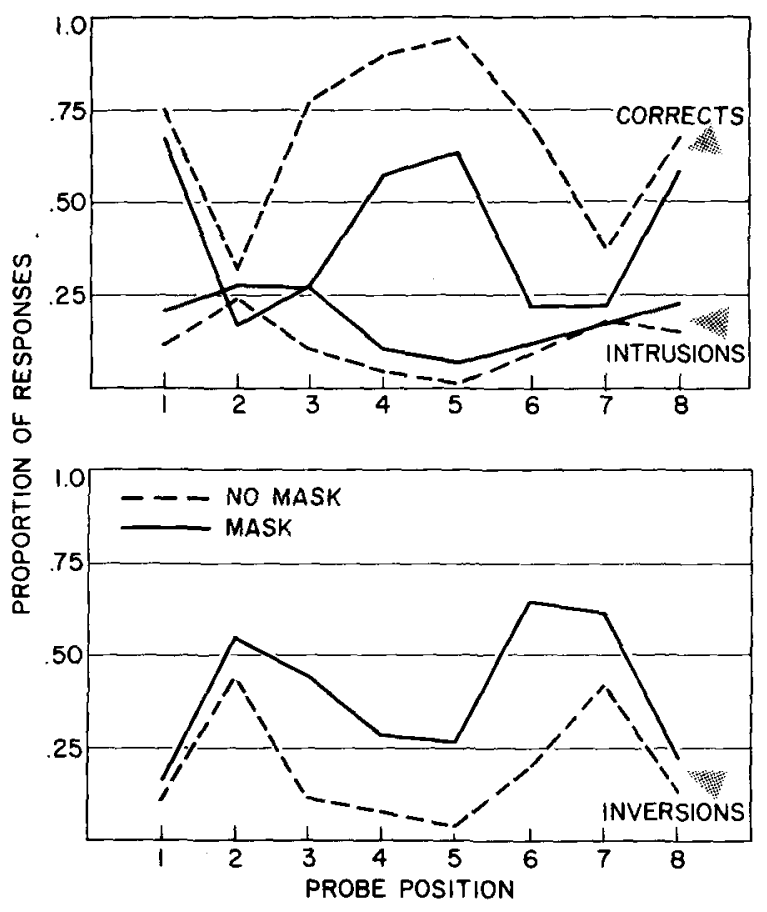

Figure 2. The top panel shows the proportion of both letters reported correctly and intrusion errors in Experiment 2 as a function of probe position and masking. The bottom panel shows the inversion errors in Experiment 2 as a function of probe position and masking.

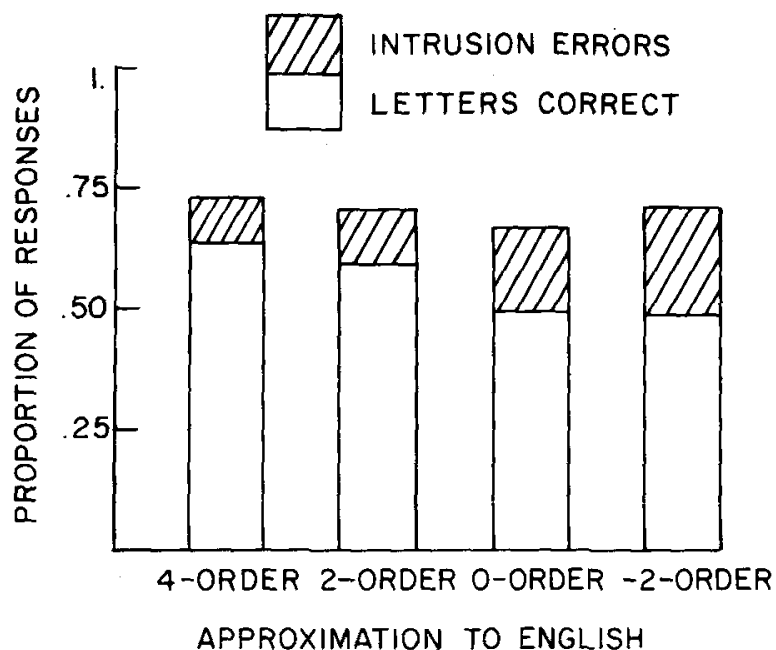

Figure 3. Proportion of both letters reported correctly and intrusion errors in Experiment 2 as a function of approximation to English.

Accuracy scores. As is shown in the top panel of Figure 2, accuracy of report took the W shape characteristic of probe studies, a pattern reflected in the overall probe-position effect, $F(7,105)=48.30$, $\mathrm{p}<.001$. In addition, masking reduced accuracy of report, $F(1,15)=108.43, p<.001$, but the reduction was greater for central items than for the end ones. The latter pattern was reflected in the interaction of probe position with masking, $F(7,105)=5.91$, $\mathrm{p}<.001$. In short, the accuracy-of-report data showed a typical selective-masking effect. Finally, as shown in Figure 3, accuracy of report increased with increases in the level of approximation to English, $F(3,45)=12.78, p<.001$. None of the other interactions, in particular the interactions involving kind of material, approached significance.

Intrusion errors. Intrusions, shown in the top panel of Figure 2, were more frequent at the ends of the array than in the middle $F(7,105)=7.74$, $\mathrm{p}<.001$. Further, masking increased the frequency of intrusions, $F(1,15)=25.95, p<.001$. Finally, as shown in Figure 3, intrusions increased as the level of approximation to English decreased, $F(3,45)=$ $10.06, p<.001$. The latter trend represents a tradeoff with accuracy of report; the product-moment correlation coefficient relating mean accuracy and mean number of intrusions was -.94 across the four levels of approximation to English. None of the other interactions approached significance.

Inversion errors. As in the previous experiment, the bulk of the errors were inversions $(65.72 \%$ of the errors, $29.7 \%$ of the responses), and as shown in the bottom panel of Figure 2, the inversions were a mirror image of the accuracy scores. Analysis of the inversions showed main effects of masking and probe position, $\mathrm{F}(1,15)=104.91, \mathrm{p}<.001$, and 
$\mathrm{F}(7,105)=32.02, \mathrm{p}<.001$, respectively. In addition, the interaction of masking with probe position was significant, $F(7,105)=5.44, p<.001$, but no other effects were significant.

Of the inversion errors, the most frequent $(57.8 \%)$ were adjacency errors, i.e., responses involving the letter adjacent to the letter probed. Inversions also occurred, however, at greater distances.

To consider the extent of spatial confusion, an error-distance analysis was conducted. For each inversion error, the distance from the correct letter position was calculated, and the frequency associated with each distance was computed separately for all subjects and conditions. Because the error distance is a function of the position probed-a distance of 1 is possible for all positions, but a distance of 7 is possible only when the end items are probedthe distance distribution is biased in favor of the shorter distances. To consider the bias, the distance distribution was compared against chance; the chance score was calculated by considering the possible errordistance frequencies. For the calculation, each inversion error was considered to contribute equally to all the distances possible at its probe position. Hence, the chance distribution of distances took into account the relative frequency at each distance.

Figure 4 shows inversion errors as a function of distance from the probed item; the scores are expressed as a deviation from chance. If inversion errors simply reflect a failure of identification, the distribution of inversions should have followed chance. As shown in the figure, however, adjacency errors were considerably more frequent than chance would predict. Masking increased intrusion errors and brought the distribution of inversions closer to chance, but as is clear in the figure, adjacency errors remained considerably more frequent than chance.

For statistical analysis, the data presented in Figure 4 were summarized by calculating the average error distance; the raw error frequencies at each error distance were converted to proportions and weighted by the error distances 1 through 7 . The expected (chance) error distance was calculated from the possible frequencies. The average error distance was 1.93 letter positions less than chance. Masking increased the error distance (relative to chance) by $.497, \mathrm{~F}(1,15)=114.62, \mathrm{p}<.001$. Thus, masking not only encouraged inversions, but also induced more distant inversions.

\section{Discussion}

The pattern of results provides clear evidence favoring an interpretation in terms of spatial localization factors over the ends-first view. The ends-first idea explains the selective-masking effect in terms of the order in which the items are identified. According to the theory, the mask is relatively more effective

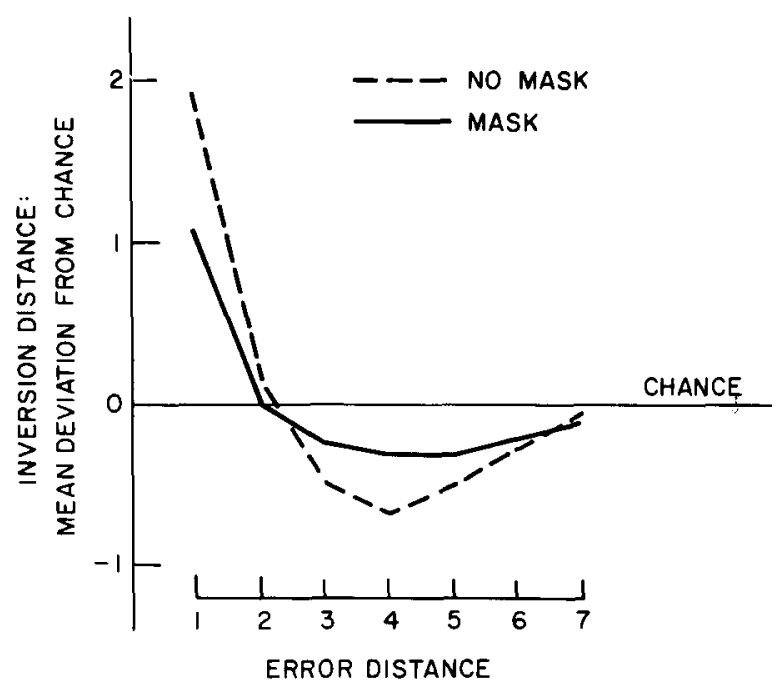

Figure 4. Inversion errors in Experiment 2 as a function of error distance. The data are expressed as a deviation from chance.

in the middle of the array because central items are identified later. Intrusions reflect a clear failure of identification processes. Following the logic of endsfirst theory, such errors should have been concentrated in the center of the array, and masking should have increased the concentration. Instead, the intrusions were concentrated at the ends. In addition, as in the first experiment, the bulk of the errors were failures of localization, and such errors were inversely related to corrects. Thus, the pattern of errors is not consistent with the theory. Rather, the interrelated pattern of errors suggests that subjects use the ends as spatial anchors. Further, given the effects of the mask on both the frequency and distance of inversions, it appears that masking disrupts spatial clarity, but that the disruption is less at the ends. Thus, in contrast to the sequential-identification idea, we suggest that the selective-masking effect reflects a relatively smaller loss of spatial clarity at the ends of the array than at the middle.

Although the localization interpretation seems straightforward, evidence reported by Lowe (1975) complicates the situation. According to Lowe, a single-bar-probe study should not show selective masking, a proscription which includes both of the present studies.

Lowe's point is based on four interrelated experiments. The first experiment involved both mask and no-mask conditions and used a single bar-probe technique. At face value, the experiment is similar to those reported here, but it did not yield selective masking. To explain the failure of the selectivemasking effect, Lowe stated that earlier studies showing the effect did not impose spatial demands but required subjects to report only the identity of the material. ${ }^{3}$ A probe study, however, requires sub- 
jects to preserve spatial information as well as identity information. As a result, he argued that the mask in a probe study may have upset location information, a possibility which could explain the failure to obtain a selective-masking effect. Accordingly, he examined the localization question with two short experiments thought to assess spatial resolution (Experiments 2 and 3 ).

In Lowe's second and third experiments, the stimuli were predictable (the digits 1 to 7 in order) and the display was followed by a mask extending across the whole visual field. Experiment 2 used a standard single-bar probe, but Experiment 3 involved a multiple-bar technique. For the latter, each stimulus position was indicated by a short bar, and the probe position was indicated by a longer bar. With the single probe (Experiment 2), accuracy of report took an inverted-U shape; with the multiple-probe technique (Experiment 3), performance across the array varied over a $30 \%$ range but was flat statistically.

From the second experiment, Lowe argued that the single-probe technique was deficient because it did not preserve spatial information, a conclusion explaining the failure of selective masking in his first experiment. From the third experiment, however, Lowe argued that the multiple-probe technique preserves spatial information but that it does so without introducing spatial anchors. Thus, he states that with the multiple-probe technique "recall performance will be determined principally by the amount of material actually processed rather than subjects' differential ability to employ the PR [partial report] cues at different positions of the arrays"' (p. 312).

Satisfied that the multiple-probe technique eliminates localization problems, Lowe used the technique to reevaluate the ends-first idea. His fourth experiment used the multiple-probe technique and found a typical selective-masking effect, a result he took as evidence for the ends-first idea.

Lowe's discussion of the failure to show selective masking (Experiment 1) focused on the adequacy of spatial information. The argument acknowledges the important role of spatial localization in the bar-probe task, and would appear to support the position urged here; namely, the selective-masking effect reflects spatial-localization factors, not ends-first identification. Lowe, however, extended the argument farther. From his second and third experiments, he claimed that the multiple-probe technique excludes spatiallocalization processes. In effect, he concludes that spatial information is necessary-a point acknowledged by his account for the failure of selective masking - but that the multiple-cue technique makes spatial information complete and equally salient at all probe positions.
Lowe's extension to the argument is troublesome. Given both the well-known limits to linear resolution (see Garner, 1962, pp. 68-69) and a task imposing a three-bit discrimination, it is unlikely that spatial information can be as complete as his account requires. Further, Lowe's evidence is less than definitive. His argument rests on the estimate of spatial clarity provided by the predictable-stimulus multipleprobe task (Experiment 3), and the evidence for the argument rests on proof of the null hypothesis. The data, however, appear to be somewhat noisy, a point reflected in the contrast between the statistical analysis and the spread of the means across a $30 \%$ range. Accepting the null hypothesis is always risky, but it appears to be particularly dangerous in this case. Second, Lowe examined only the mask condition. The selective-masking effect is a relative one; it concerns the relative accuracy across the array for mask and no-mask conditions. Thus, the estimate of spatial salience should have been a relative one; Lowe should have tested both the mask and the no-mask conditions. Finally, the data address only part of the problem. Even if the multiple-probe technique were to ensure that all probes were equally easy to identify, the task does not deal with spatial clarity within the stimulus array itself.

Aside from such difficulties, Lowe's report raises an interesting paradox. Our first two experiments have shown a straightforward selective-masking effect with a single-probe technique. Lowe's first experiment, however, failed to show the effect with the single probe. If Lowe's arguments about the single-probe are correct, why did our single-probe studies, like his fourth experiment, show selective masking?

The paradox can be resolved by considering some features of Lowe's procedures. In his first experiment, the masking stimulus extended slightly beyond the letter array. Under such circumstances, changing from the letter array to the mask is likely to have induced an apparent-motion effect. More important, a mask extending across the entire visual field should disrupt the anchor effect provided by the end items (cf. Haber \& Standing, 1969). Curiously, although comparison among the tasks is required for his arguments, Lowe's experiments include both kinds of mask; the two predictable-stimulus tasks (Experiments 2 and 3) used a mask extending across the whole visual field, but the other experiments (Experiments 1 and 4) used a mask extending past the letter array but not across the whole field.

We suggest that his failure to find a selectivemasking effect with the single-probe technique (Experiment 1) reflects the use of a mask which disrupts spatial clarity at the ends of the array. Similarly, the return of the effect with the multiple- 
probe procedure (Experiment 4) likely reflects the spatial information provided by the multiple probes. Our interpretation is based on the spatial-anchor idea, and, consistent with the interpretation, our experiments, like that reported by Merikle and Glick (1976), used a row of mask characters positioned to cover each letter without extending beyond the array. Such a mask should preserve spatial information in the same way the multiple probes preserved the end anchors in Lowe's fourth experiment.

\section{EXPERIMENT 3}

Our interpretation of the empirical paradox assumes that, unless special precautions are taken, a mask extending beyond the stimulus disrupts the spatial anchors normally provided by the ends. The third experiment was designed to support the interpretation. In the experiment, subjects were shown a predictable stimulus followed by a single bar probe under both mask and no-mask conditions. Two lengths of mask were used. The short mask covered the stimulus exactly, and the long mask extended two character spaces on either side of the stimulus. If the interpretation is correct, performance on the long mask should take an inverted-U shape reflecting loss of the end anchors. With the short mask, however, performance at the ends should be high, a pattern reflecting the use of the end anchors.

\section{Method}

Subjects. The subjects were 20 undergraduates from the same pool as in the previous experiment. None had participated in either of the previous experiments.

Apparatus and materials. The apparatus was the same as that used in the previous experiments. The stimulus was constant and consisted of the digits 1 through 8 in sequence. The mask involved either 8 or 12 characters. When shown on the screen, the 12-character mask covered each digit and extended two positions on either side of the digit string. The eight-character mask covered the digits but did not extend past the digit string. The visual angle of the extended mask was approximately $4^{\circ} 39^{\prime}$ by $21^{\prime}$; the angle for the digit string and the eight-character mask remained as in the previous experiments.

Procedure. The procedure was essentially the same as in the first experiment. The exposure time for the display was $100 \mathrm{msec}$, the probe appeared for $200 \mathrm{msec}$, and when masking was involved, the probe and the mask appeared concurrently.

The instructions required the subjects to report the digit indicated by the probe, and the subjects knew that the stimulus array was constant. In addition, the subjects were told the digit 1 on the screen occupied the leftmost position, and so forth.

Design. Each subject received 144 trials, nine replicates for the factorial combination of probe position and masking. Each position was probed equally often in the mask and no-mask conditions. The order in which the conditions were administered was determined randomly for each subject.

Two groups of 10 subjects were tested. One group received nomask trials and trials with the eight-character mask; the other group received no-mask trials and trials with the 12-character mask.

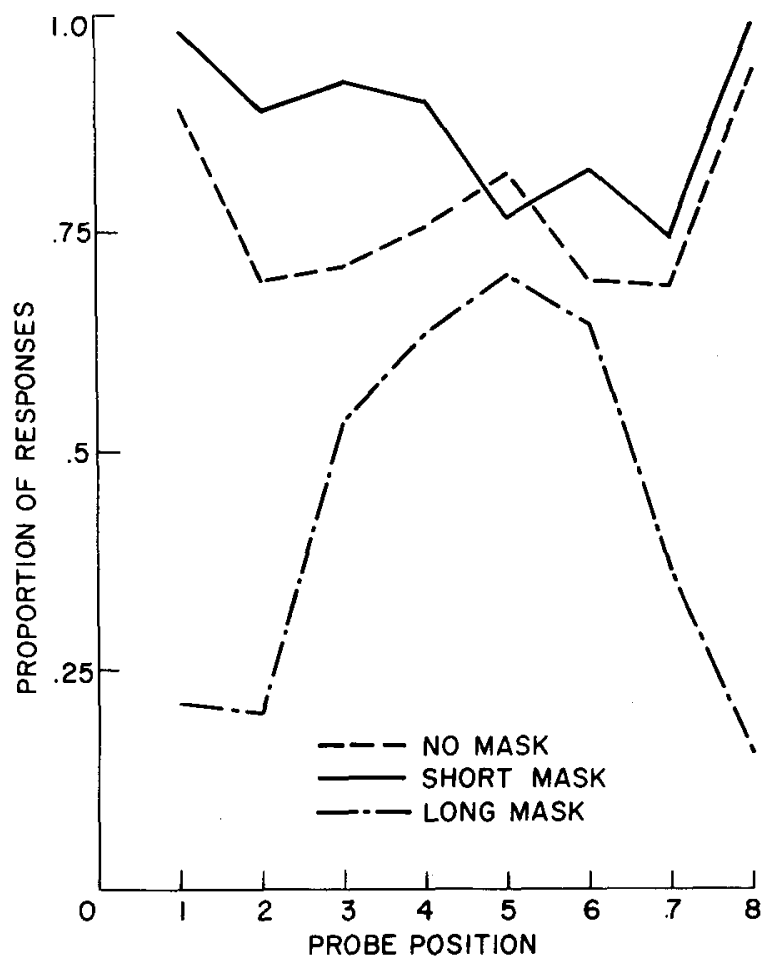

Figure 5. Proportion of items reported correctly in Experiment 3 as a function of probe position and masking condition.

\section{Results}

The results are summarized in Figure 5. Each group received both mask and no-mask cases. Because an analysis of variance for the no-mask cases indicated that the kind of mask did not influence performance on the no-mask trials, the no-mask data from the two groups were combined. Thus, the figure shows accuracy of report as a function of probe position for the no-mask, short-mask, and long-mask conditions.

As is shown in the figure, performance with no mask took a $U$ shape with a local peak in the center, $F(7,126)=4.91, p<.001$. The no-mask data illustrate the use of spatial anchors and confirm an earlier report by Townsend (1973, Experiment 3). She used an unpredictable stimulus but asked subjects to ignore it and to report the location of a single bar probe.

The short mask yielded a $U$ shape across the digit array, $F(7,63)=3.46, p<.01$. In contrast, the long mask showed an inverted $U$ shape, $F(7,63)=11.24$, $\mathrm{p}<.001$. There was, of course, a large interaction of Mask Length by Probe Position, $F(7,126)=11.82$, $\mathrm{p}<.001$. Finally, performance with the short mask was considerably better than with the long mask, $\mathrm{F}(1,18)=84.54, \mathrm{p}<.001$.

The results confirm the interpretation introduced 
earlier. A mask extending beyond the ends of the array disrupts the end anchors, but a mask covering the letters without extending past the ends maintains the anchor effect.

\section{CONCLUSIONS}

The ends-first view attributes selective masking to a sequential character-identification strategy. In contrast, we suggest that performance in the bar-probe task largely reflects spatial addressing processes. To support our view, we have presented three kinds of evidence. In the first experiment, the role of spatial localization was exposed by using words to minimize the contribution of identification factors. Nevertheless, the task yielded a typical selective-masking effect, a result contrary to the sequential-identification view but consistent with the localization explanation. The second experiment used a more standard pseudoword task and included an extensive analysis of errors. Again, a standard selective-masking effect appeared, but the pattern of errors favored the localization view. Contrary to the ends-first view, for example, clear failures of identification were concentrated at the ends. Also, the bulk of the errors represented mislocations, and masking increased the mislocation distance. The third experiment addressed the issues indirectly by testing predictions derived from the localization view in response to inconsistencies posed by the sequential-identification idea.

All three experiments concern the way subjects use spatial information. The bar probe is, in effect, a spatial instruction, and the data suggest that for such tasks an attentional process uses the instruction to focus on selected portions of the array. Presumably, the mechanism also uses the spatial information within the array, and its success depends on the salience of the joint information. Unfortunately, the present data do not offer much guidance concerning the nature of the attentional process. Consistent with Estes' (1975) analysis, however, there were fewer inversion errors in the first experiment, which used words, than in the second, which used pseudowords. Similarly, inversions in the word experiment were more likely to be adjacent than in the pseudoword case. To put the process into a rough perspective, we offer some speculations about its relation to processes suggested in another context.

Building from a series of free-recall studies using pseudowords, Mewhort and Beal (1977) have suggested a model for word identification which requires a postidentification character buffer. The model, like that of Smith and Spoehr (1974), implicates a scanparse operation which reads the buffer, forms a series of verbal-temporal units, and passes the units into short-term memory. The scanning mechanism, an overlearned reading routine, represents a kind of spatial-integration mechanism. We suggest that the attentional process implicated in the present study also addresses the character buffer. The two mechanisms, however, are quite different; they serve different ends and offer specialized resources admirably suited for the different tasks. In free recall, for example, short-term memory provides the major limit to performance, and to overcome the limit, the scan-parse operator prepares the material in a fashion suitable for short-term memory processes. In a bar-probe task, however, the implicit instructions (the task demands) require subjects to keep each letter as uistinct as possibie, a strategy permitting independent addressing of particular letters. Thus, in the context of the scanning model, we view the attentional process and the scan-parse operator as alternate processing routines "called" by the demands of the task. Such a position views simple tachistoscopic tasks in terms of a problem-solving analogy; various processing strategies represent solutions to the implicit (and explicit) instructions given to the subjects. The position also acknowledges a gap in current theory; future work should include an explicit account of how processing routines are assigned to particular tasks.

\section{REFERENCE NOTE}

1. Mewhort, D. J. K. DIS: An n-channel tachistoscope algorithm. Unpublished manuscript, Queen's University, 1978.

\section{REFERENCES}

BRYdEN, M. P. A model for the sequential organization of behaviour. Canadian Journal of Psychology, 1967, 21, 37-56.

Butter, B. Selective attention and target search with brief visual displays. Quarterly Journal of Experimental Psychology, 1975, 27, 467.477.

Butler, B. E.. \& Merikle, P. M. Selective masking and processing strategy. Quarterly Journal of Experimental Psychology, 1973, 25, 542-548.

Dick, A. O. Iconic memory and its relation to perceptual processing and other memory mechanisms. Perception \& Psychophysics, 1974, 16, 575.596.

Eriksen, C. W., \& Rohrbaugh, J. W. Some factors determining efficiency of selective attention. American Journal of Psychology, 1970, 83, 330-342.

Estes, W. K. Memory, perception, and decision in letter identification. In R. L. Solso (Ed.), Information processing and cognition: The Loyola symposium. Hillsdale, N.J: Erlbaum, 1975.

GARNER, W. R. Uncertainty and structure as psychological concepts. New York: Wiley, 1962.

Haber, R. N., \& Standing, L. Location of errors with a post-stimulus indicator. Psychonomic Science, 1969, 17, 345-346.

Henderson, L., \& Park, N. Are the ends of tachistoscopic arrays processed first? Canadian Journal of Psychology, 1973, 27. 178-183.

Hirata, K.. \& Bryden, M. P. Tables of letter sequences varying in order of approximation to English. Psychonomic Science, 1971, 25, 322-324.

KuČEra, H., \& Francis, W. N. Computational analysis of 
present-day American English. Providence, R.I: Brown University Press, 1967.

LowE, D. G. Processing of information about location in brief visual displays. Perception \& Psychophysics, 1975, 18, 309-316.

MatThews, M. L. Locus of presentation and the selective masking effect. Canadian Journal of Psychology, 1973, 27, 343-349.

Mayzner, M. S., \& Tresselt, M. E. Tables of single-letter and digram frequency counts for various word-length and letterposition combinations. Psychonomic Monograph Supplements, 1965,1 (2, Whole No. 2), 13-32.

Merikif, P. M. Selective backward masking with an unpredictable mask. Journal of Experimental Psychology, 1974, 103, 589-591.

Merikle, P. M., \& Coltheart, M. Selective forward masking. Canadian Journal of Psychology, 1972, 26, 296-302.

Merikle, P. M., Coltheart, M., \& Lowe, D. G. On the selective effects of a patterned masking stimulus. Canadian Journal of Psychology, 1971, 25, 264-279.

Merikle, P. M., \& Glick, M. J. Processing order in visual perception. Quarterly Journal of Experimental Psychology, $1976,28,17-26$.

Mewhort, D. J. K. Accuracy and order of report in tachistoscopic identification. Canadian Journal of Psychology, 1974. 28, 383-398.

Mewhort, D. J. K., \& BeAl, A. L. Mechanisms of word identification. Journal of Experimental Psychology: Human Perception and Performance, 1977, 3, 629-640.

Smith, E. E., \& SPOEHR, K. T. The perception of printed English: A theoretical perspective. In B. H. Kantowitz (Ed.), Human information processing: Tutorials in performance and cognition. Hillsdale, N.J: Erlbaum, 1974.

SNYDER, C. R. R. Selection, inspection, and naming in visual search. Journai of Experimental Psychology, 1972, 92, 428-431.

Townsend, V. M. Loss of spatial and identity information following a tachistoscopic exposure. Journal of Experimental Psychology. 1973, 98, 113-118.

\section{NOTES}

1. An ascending-descending method of limits was used to determine an approximate threshold for reading eight-letter words. On each trial, subjects saw a different word followed immediately by a mask for $150 \mathrm{msec}$. The exposure time for the words was varied to determine the threshold. The mean exposure time averaged across 16 subjects was approximately $35 \mathrm{msec}(\mathrm{SD}=7.5$ ). The number of trials required to establish a stable threshold varied between 30 and 50 .

2. The experiment has been replicated with small changes of procedure. For the replication, the exposure duration was $150 \mathrm{msec}$ and the probe appeared for $150 \mathrm{msec}$. Whep relevant, the mask appeared concurrently with the probe. The pattern of results was virtually identical to that reported here. Thus, it appears that variation in probe duration over the range described does not alter the results dramatically. Using a larger variation, however, Townsend (1973) reported better performance with a short probe $(40 \mathrm{msec})$ than with a long one $(900 \mathrm{msec})$.

3. It is curious that Lowe would attempt such an argument; he cited a probe study by Merikle and Coltheart (1972) which included both a spatial and an identification component and which showed a clear selective-masking effect. More important, his argument raises a fundamental theoretical point: To what extent can one infer from a description of a task, the nature of the processes involved in executing it? We suggest that such inferences are not straightforward. For example, the studies Lowe claims do not involve spatial information include the free-recall cases reported by Merikle et al. (1971). While the free-recall task does not require subjects to preserve spatial information, it is likely that they use it in preparing an ordered report (e.g., Bryden, 1967; Mewhort, 1974).

(Received for publication February 20, 1978; revision accepted June 1,1978 .) 